\title{
Intratracheal administration of isosorbide dinitrate improves pulmonary artery pressure and ventricular remodeling in a rat model of heart failure following myocardial infarction
}

\author{
XUELIAN WANG*, QINGQING XU*, TIANQI LI, YAOCONG RONG, \\ WEILIN HONG, YAN HUANG and XINGUI GUO \\ Department of Cardiology, Huadong Hospital Affiliated to Fudan University, Shanghai 200040, P.R. China
}

Received June 17, 2016; Accepted April 21, 2017

DOI: $10.3892 /$ etm.2017.4707

\begin{abstract}
Pulmonary hypertension due to left heart disease is associated with poor outcomes. This study investigated the beneficial effects of isosorbide dinitrate (ISDN) inhalation on pulmonary pressure and ventricular remodeling in a rat model of heart failure (HF) following myocardial infarction (MI). To assess the effect of ISDN on pulmonary pressure, 20 male Sprague-Dawley (SD) rats were randomized to four groups: Normal saline (NS) $1 \mathrm{ml} / \mathrm{kg}$, ISDN $1 \mathrm{mg} / \mathrm{kg}$, NS $3 \mathrm{ml} / \mathrm{kg}$ or ISDN $3 \mathrm{mg} / \mathrm{kg}$ following coronary ligation. Assessments included pulmonary and systemic artery pressure alterations, lung weight/body weight and plasma nitric oxide (NO) concentration. To assess the effect of ISDN on ventricular remodeling, 30 SD rats were randomized to three groups: Sham surgery, MI-NS (intratracheal NS $3 \mathrm{ml} / \mathrm{kg}$ for 13 days following coronary ligation), and MI-ISDN (intratracheal ISDN $3 \mathrm{mg} / \mathrm{kg}$ for 13 days following coronary ligation). On day 15, all rats underwent echocardiogram and hemodynamic assessments. The area affected by MI was evaluated using microscopy and vascular endothelial growth factor (VEGF) expression was examined using immunohistochemistry. Plasma epinephrine, norepinephrine and brain natriuretic peptide (BNP) levels were assessed by ELISA. Intratracheal ISDN reduced pulmonary and systematic artery pressure without pulmonary edema when compared with NS. The reduction was associated with increased plasma NO levels. ISDN inhalation for 14 days reduced MI size and alleviated left and right ventricular remodeling following MI. These hemodynamic and morphological improvements were associated with decreased plasma
\end{abstract}

Correspondence to: Dr Tianqi Li, Department of Cardiology, Huadong Hospital Affiliated to Fudan University, 221 West Yan'an Road, Shanghai 200040, P.R. China

E-mail: leeheng78@sina.com

${ }^{*}$ Contributed equally

Key words: pulmonary hypertension, isosorbide dinitrate, ventricular remodeling, cardiac function, heart failure, myocardial infarction epinephrine, norepinephrine and BNP levels, and an increased VEGF positive area at the border of MI region. In conclusion, intratracheal administration of ISDN was effective in improving ventricular remodeling and cardiac function in a rat model of HF following MI.

\section{Introduction}

Myocardial infarction (MI) is the most common cause of mortality for patients with cardiovascular diseases (1). Elevation of left ventricular (LV) filling pressures following MI may lead to secondary pulmonary hypertension $(\mathrm{PH})$ and ventricular remodeling, and even heart failure (HF) (2). LV failure causes $\mathrm{PH}$ and increased right ventricular (RV) afterload, leading to RV remodeling and dysfunction (3). $\mathrm{PH}$ due to left heart disease (LHD) mainly arises from left heart systolic or diastolic dysfunction, or valvular heart disease, and is associated with poor clinical outcome (4). Cardiac remodeling following MI involves several molecular and cellular mechanisms in the ischemic and non-ischemic myocardium (5). The persistently elevated pulmonary pressures may deteriorate endothelial dysfunction, decrease nitric oxide (NO) availability and increase endothelin expression $(2,6)$. PH associated with LHD may be reversible in the early stages, while long-standing $\mathrm{PH}$ may be irreversible due to cardiac remodeling (7).

The guidelines for PH generally discourage the use of drugs approved for $\mathrm{PH}$ reduction to treat $\mathrm{PH}$ due to LHD as evidence to support their use is lacking (8). However, the guidelines give little advice on the management of excessive vasoconstriction or remodeling of the pulmonary vasculature in reactive $\mathrm{PH}$. Therefore, it is important to develop and evaluate novel therapies directly affecting the pulmonary vascular component.

NO is a small gaseous molecule produced by endogenous NO synthases that is involved in the regulation of vascular tone and blood pressure, in angiogenesis and in endothelial integrity $(9,10)$. Reduced NO bioavailability is associated with a number of cardiovascular diseases (11-13), including $\mathrm{PH}$. Therefore, the use of exogenous NO is important in the treatment of PH following LHD (14,15). NO donors such as organic nitrates (for example, nitroglycerin) have been used for over a century; however, the development of tolerance is a major problem with these drugs (16). 
Inhalation of NO during myocardial ischemia may alleviate secondary pulmonary hemodynamic dysfunction, reduce infarct size and improve cardiac function (14). In the last decade, studies have shown that inhalation of nebulized nitrates is able to alleviate $\mathrm{PH}$, whereas the effects of $\mathrm{NO}$ on ventricular remodeling and cardiac function are poorly understood $(17,18)$. Isosorbide dinitrate (ISDN) is an NO donor that prevents LV remodeling and degradation of cardiac function following MI (19,20).

However, there is a lack of supportive management of excessive vasoconstriction or remodeling of the pulmonary artery in $\mathrm{PH}$ following MI. Therefore, the present study investigated the benefits of intratracheal ISDN instillation in the amelioration of pulmonary pressure and ventricular remodeling in a rat model of $\mathrm{HF}$ following MI.

\section{Materials and methods}

Study design. The study design and the protocol are presented in Fig. 1. The study comprised two parts. The first part examined the effect of ISDN instillation on pulmonary pressure, and the second part examined the effect of ISDN instillation on ventricular remodeling.

Animals. The study was conducted on 50 male juvenile Sprague-Dawley (SD) rats (5-6 weeks old), with a body weight (BW) of 200-250 g, which were purchased from the Shanghai Laboratory Animal Centre (CAS; Shanghai, China). A standard pellet diet and water were provided ad libitum. The animals were housed in a temperature- $\left(21-23^{\circ} \mathrm{C}\right)$ and humidity-controlled (40-70\%) room and maintained on a 12-h light/dark cycle. The study protocol was approved by the Animal Research Ethics Committee of Huadong Hospital Affiliated to Fudan University (Shanghai, China). All animals received care in accordance with the Guidance for the Care and Use of Laboratory Animals (21).

MI rat model. The MI model has been described previously (22). In brief, rats were anesthetized with chloral hydrate $[0.3 \mathrm{~g} / \mathrm{kg}$, intraperitoneally (i.p.)] and placed in a supine position. The chest wall was shaved. A left thoracotomy was performed at the fourth intercostal space. Respiration was maintained by mechanical ventilation following orotracheal intubation. Following exposure of the heart, the middle left ascending coronary artery was ligated, $\sim 4 \mathrm{~mm}$ below the anterior-inferior edge of the left atrium. The local myocardium became white immediately following the ligation due to MI. The thorax was closed and the rats recovered from anesthesia. Electrocardiograms were recorded prior to and following surgery. Successful establishment of this model was defined by a significant ST segment elevation. The rats received 400,000 $\mathrm{U} / \mathrm{kg}$ penicillin intramuscularly immediately following the surgery and daily for 3 days.

Mean pulmonary arterial pressure (MPAP) and mean arterial pressure (MAP) measurements subsequent to ISDN or normal saline (NS) instillation in the trachea. The pharmacological effects of intratracheal ISDN instillation were observed. Twenty successful rat models were randomized in the following four groups (each $\mathrm{n}=5)$ : NS ( $1 \mathrm{ml} / \mathrm{kg}$ ) group, ISDN
(1 ml/kg) group, NS (3 ml/kg) group and ISDN $(3 \mathrm{ml} / \mathrm{kg})$ group. Polyvinyl catheters (internal diameter, $0.7 \mathrm{~mm}$ ) were directly inserted into the left carotid artery and right jugular vein. When hemodynamic data were stable for $15 \mathrm{~min}$ after ligation, NS or ISDN was instilled into the trachea of the MI model rats (23). For rats in the NS $(1 \mathrm{ml} / \mathrm{kg})$ or ISDN $(1 \mathrm{ml} / \mathrm{kg})$ groups, $1 \mathrm{ml} / \mathrm{kg} \mathrm{NS}$ or $1 \mathrm{mg} / \mathrm{kg}$ ISDN (batch no. 555600; UCB Pharma GmbH, Monheim am Rhein, Germany) was instilled into the trachea. In the NS (3 ml/kg) group, $1 \mathrm{ml} / \mathrm{kg}$ NS was instilled thrice at 5-min intervals to provide a total dose of $3 \mathrm{ml} / \mathrm{kg}$. In the ISDN ( $3 \mathrm{ml} / \mathrm{kg}$ ) group, ISDN was administered using the protocol described for the NS $(3 \mathrm{ml} / \mathrm{kg})$ group. The dosage regimen and methodology were based on a pilot study demonstrating that the instillation of three $1 \mathrm{mg} / \mathrm{kg}$ doses of ISDN at 5-min intervals achieved the desired effects without causing adverse effects such as hypotension or sudden death (data not shown). MPAP and MAP were measured using a specialized system (MFLab3.01; Shanghai Jia Long Educational Instrument Factory, Shanghai, China) (24). Changes in MPAP $(\triangle \mathrm{MPAP} \%)$ and in MAP $(\triangle \mathrm{MAP} \%)$ were calculated using the following formulae and compared among the groups: $\triangle \mathrm{MPAP}_{\mathrm{x} \text { min }}=\left(\mathrm{MPAP}_{\mathrm{x} \text { min }}-\mathrm{MPAP}_{0 \text { min }}\right) / \mathrm{MPAP}_{0 \text { min }} \mathrm{x} 100$; $\Delta \mathrm{MAP}_{\mathrm{x} \text { min }}=\left(\mathrm{MAP}_{\mathrm{x} \text { min }}-\mathrm{MAP}_{0 \text { min }}\right) / \mathrm{MAP}_{0 \text { min }} \mathrm{x} 100$.

Lung weight/BW ratio and plasma NO measurement. Following the measurement of MPAP and MAP, the lungs of rats in the NS $(3 \mathrm{ml} / \mathrm{kg})$ and ISDN ( $3 \mathrm{ml} / \mathrm{kg})$ groups were dissected and weighed. The ratios of wet lung weight to BW, dry lung weight to BW, and dry to wet lung weight were calculated.

Blood samples were taken from rats in the NS $(3 \mathrm{ml} / \mathrm{kg})$ and ISDN ( $3 \mathrm{ml} / \mathrm{kg}$ ) groups immediately following the final NS or ISDN instillation. Blood samples were centrifuged $3,000 \mathrm{x} \mathrm{g}$ for $10 \mathrm{~min}$ at $4^{\circ} \mathrm{C}$ and the supernatant was used to assess plasma NO levels using an NO assay kit (cat. no. A012; Nanjing Jiancheng Bioengineering Institute, Nanjing, China). In order to determine the immediate effects of ISDN, NO was measured from blood samples taken immediately following ISDN/NS instillation.

ISDN/NS inhalation in rats following MI. In the second part of the study, on day 0,30 SD rats were randomized into three groups (each $n=10$ ): MI-NS group, MI-ISDN group and sham group. On day 1 , coronary ligation was performed on the rats in the MI-NS and MI-ISDN groups, as previously described (22). When hemodynamic stability was achieved 15 min after ligation, $1 \mathrm{ml} / \mathrm{kg}$ NS was instilled intratracheally every $5 \mathrm{~min}$ thrice to provide a total dose of $3 \mathrm{ml} / \mathrm{kg}$ in the MI-NS group. In the MI-ISDN group, $3 \mathrm{mg} / \mathrm{kg}$ ISDN was instilled intratracheally using the same protocol. The thoracic cavity of rats in the sham group was opened without coronary ligation.

For the following 13 days (days 2-14), ISDN or NS inhalation was performed in the MI-NS and MI-ISDN groups, respectively. ISDN (3 $\mathrm{mg} / \mathrm{kg})$ or NS $(3 \mathrm{ml} / \mathrm{kg})$ were nebulized using an ultrasonic nebulizer (NE105; Guangying Electronics Co., Ltd., Foshan, China) and inhaled by the rats. Inhalation was conducted for 15 min daily. Rats in the sham group did not receive any inhalation treatment.

Echocardiogram and hemodynamic measurements. On day 15 , all rats from each group were anesthetized with 
A

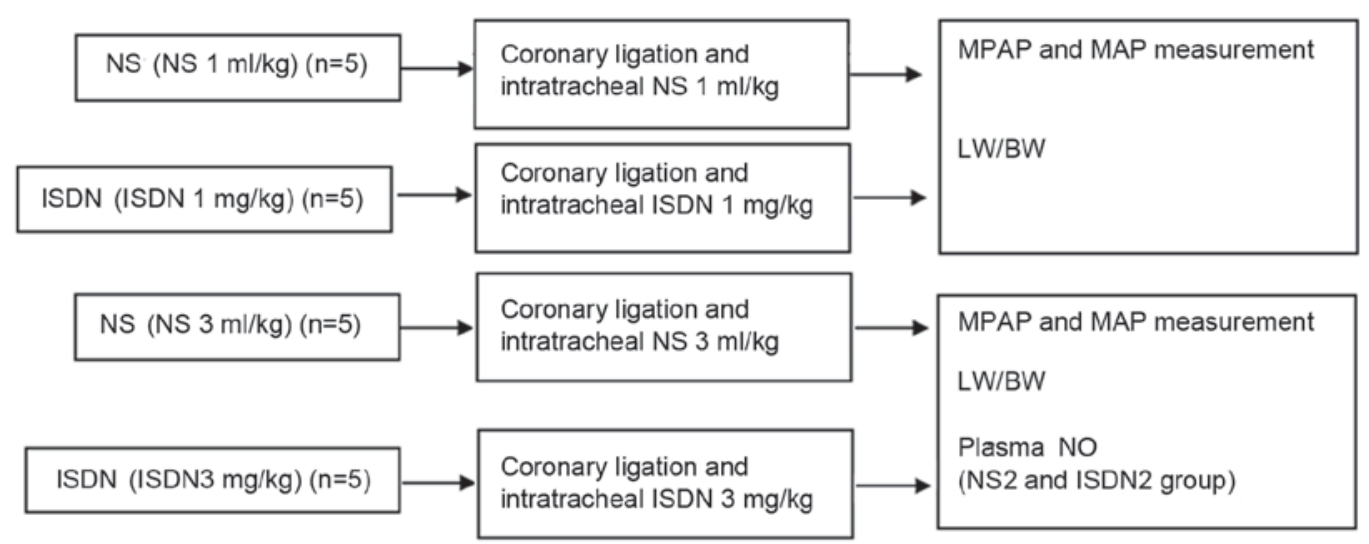

B

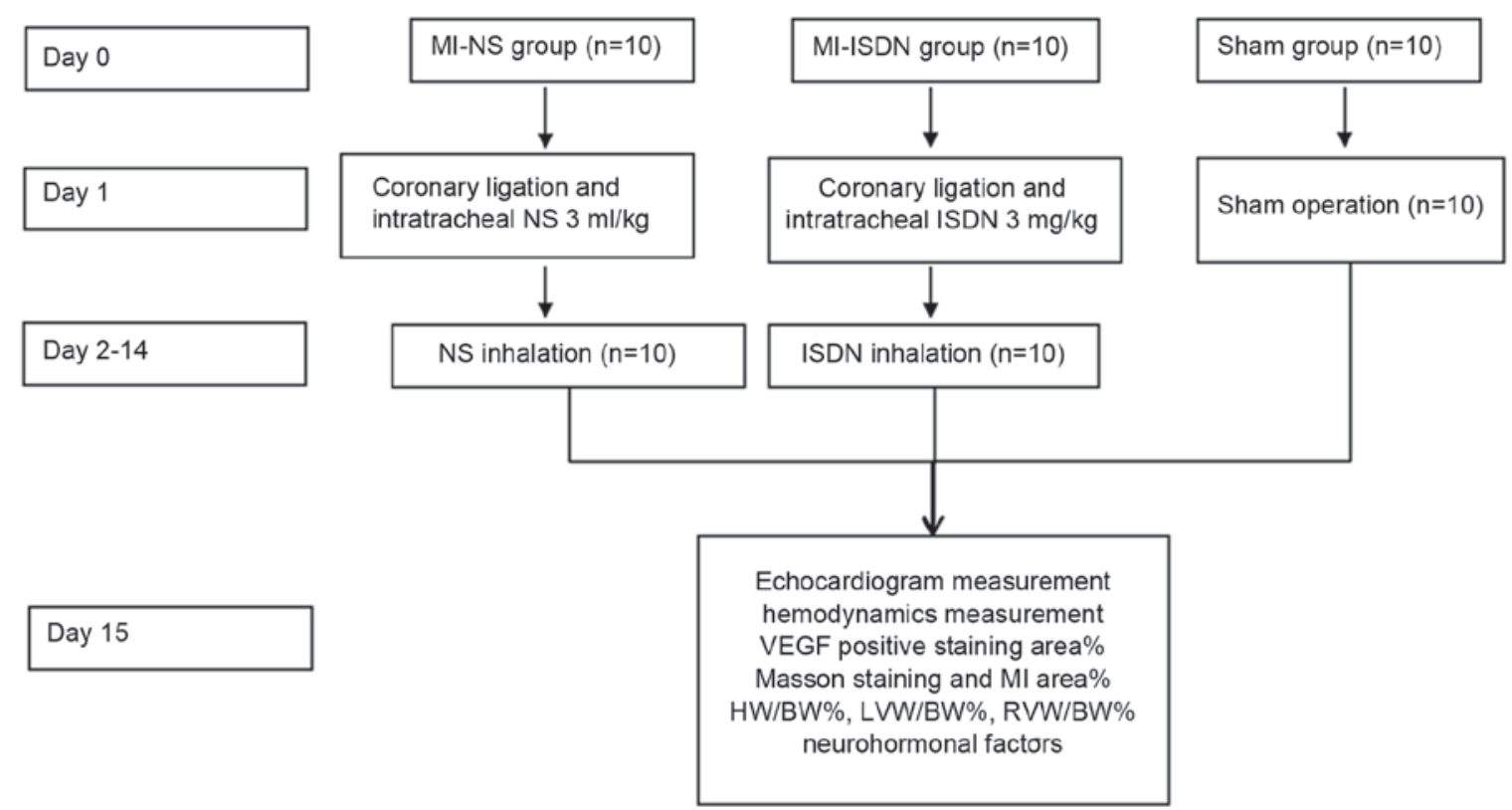

Figure 1. Study design. The study comprised two parts. (A) The first examined the effect of ISDN instillation on pulmonary pressure. (B) The second examined the effect of ISDN instillation on ventricular remodeling. NS, normal saline; ISDN, isosorbide dinitrate; MPAP, mean pulmonary arterial pressure; MAP, mean arterial pressure; LW, lung weight; BW, body weight; NO, nitric oxide; MI, myocardial infarction; VEGF, vascular endothelial growth factor.

urethane (1 mg/kg, i.p.). Echocardiogram measurements were obtained. LV internal diameter at end-diastole (LVIDd) and systole (LVIDs), LV volume at end-diastole (LV Vol d) and systole (LV Vol s), LV post wall diameters at end-diastole (LVPWd) and systole (LVPWs), LV anterior wall diameters at end-diastole (LVAWd) and systole (LVAWs), LV ejection fraction (LVEF) and fraction shortening (FS) were evaluated.

Catheters were inserted into the left carotid artery and right jugular vein to measure RV ventricular end-diastolic pressure (RVEDP), central venous pressure (CVP), LV systolic pressure, LV end-diastolic pressure (LVEDP) and the maximum rising and dropping rates of $L V$ or $R V$ pressure $\left( \pm \mathrm{dp} / \mathrm{dt}_{\max }\right)$ were evaluated and compared among the groups. All values were recorded and analyzed using an MFLab 3.01 system.

Levels of neurohormonal factors. On day 15, blood was sampled from the abdominal aorta ( 10 ml/rat). Plasma levels of B-type natriuretic peptide (BNP), epinephrine, norepinephrine and angiotensin II were assessed using ELISA kits; BNP ELISA kit (cat. no. CK-E30445R), epinephrine ELISA kit (cat. no. CK-E30233R), norepinephrine ELISA kit (cat. no. CK-E30189R), and an angiotensin II ELISA kit (cat. no. CK-E30668R; all from Biocalvin, Suzhou, China).

Heart weight $(H W) / B W \%, L V$ weight/BW\% and $R V$ weight/BW\% evaluation. On day 15 , the hearts were harvested and weighed. The HW/BW\%, LV weight/BW\% and RV $\mathrm{RVW} / \mathrm{BW} \%$ values were calculated.

Immunohistochemical staining, and estimation of MI and $V E G F$-positive area percentages. On day 15 , hearts were sliced transversely into several pieces from the basal to apex plane. Sections with thickness of $4 \mu \mathrm{m}$ were fixed in $4 \%$ paraformaldehyde at $37^{\circ} \mathrm{C}$ for $10 \mathrm{~min}$ and stained using Masson staining methods (Masson stain kit; HL70013; Nanjing Jiancheng Bioengineering Institute, Nanjing, China) according to the manufacturer's protocol and the MI area \% was calculated using the IMS Imaging system (version 2.1.1; Shanghai ShenTeng Information Technology Co., Ltd., Shanghai, China). The infarct size was determined as the mean percentage of 
the epicardial and endocardial circumference occupied by scar tissue, as observed on the stained sections using a light microscope (25).

The myocardium at the border of the MI area was immunohistochemically stained using rabbit anti-rat vascular endothelial growth factor (VEGF) antibody (cat. no. 9698; Cell Signaling Technology, Inc., Danvers, MA, USA). Tissues were dehydrated in a graded series of ethanol and fixed at $37^{\circ} \mathrm{C}$ in $4 \%$ paraformaldehyde for $24 \mathrm{~h}$ and embedded in paraffin. Paraffin-embedded tissues were sectioned at $4 \mu \mathrm{m}$ and deparaffinized. They were subjected to epitope retrieval by immersion in $0.01 \mathrm{~mol} / 1$ sodium citrate buffer (Sinopharm Chemical Reagent Co., Ltd., Shanghai, China) with pH 6.0, heated in a microwave $\left(98^{\circ} \mathrm{C}\right.$ for $\left.10 \mathrm{~min}\right)$ and allowed to cool for $20 \mathrm{~min}$. Endogenous peroxidase was inactivated with $3 \% \quad \mathrm{H}_{2} \mathrm{O}_{2}$. Samples were incubated for $120 \mathrm{~min}$ with 1:200 rabbit anti-rat VEGF antibody at $37^{\circ} \mathrm{C}$, washed and subsequently incubated with peroxidase AffiniPure Goat Anti-Rabbit immunoglobulin G (1:200; cat. no. 111-035-003; Jackson ImmunoResearch Laboratories, Inc., West Grove, PA, USA) conjugated to biotin for $30 \mathrm{~min}$ at $37^{\circ} \mathrm{C}$. The sections were rinsed with PBS, counterstained with hematoxylin at $37^{\circ} \mathrm{C}$ for $1 \mathrm{~min}$, rinsed again and mounted. Microscopic analysis was performed using a light microscope under high-power magnification (x200). The VEGF-positive area (\%) was evaluated as previously described (26) using the IMS Imaging system.

Statistical analysis. All values are presented as the mean \pm standard deviation. Values were compared using one-way analysis of variance (ANOVA) for intergroup data. The least significant difference test or Tamhane's T2 test was performed as a post hoc test following ANOVA. Data were analyzed using SPSS 17.0 software (SPSS, Inc., Chicago, Il, USA). Two-sided P-values $<0.05$ were considered to indicate a statistically significant result.

\section{Results}

Acute effects of ISDN intratreacheal administration on hemodynamics in MI rats. Following the intratracheal instillation of $1 \mathrm{mg} / \mathrm{kg}$ ISDN, MPAP and MAP were decreased at $1 \mathrm{~min}$ and returned gradually to near the baseline within $5 \mathrm{~min}$, as shown by $\triangle \mathrm{MPAP} \%$ and $\triangle \mathrm{MAP} \%$ being approximately-24 and $-34 \%$, respectively, at $1 \mathrm{~min}$, and gradually tending to return to $0 \%$ (Fig. $2 \mathrm{~A}$ and $\mathrm{B}$ ). Therefore, the dosage of $3 \mathrm{mg} / \mathrm{kg}$ of ISDN administered in a divided form ( $1 \mathrm{mg} / \mathrm{kg}$ every $5 \mathrm{~min}$ ) achieved the desired effects without significant adverse events. $\triangle \mathrm{MPAP} \%$ and $\triangle \mathrm{MAP} \%$ were significantly greater in the ISDN $(1 \mathrm{ml} / \mathrm{kg})$ group compared with the NS $(1 \mathrm{ml} / \mathrm{kg})$ group at all time points, with the exception of 5 min $(\mathrm{P}<0.05$; Fig. $2 \mathrm{~A}$ and $\mathrm{B})$. Similar results were observed in the ISDN $(3 \mathrm{ml} / \mathrm{kg})$ and NS $(3 \mathrm{ml} / \mathrm{kg})$ groups (Fig. 2C and D), where the 5-min cycles were clearly observed.

Lung weight/BW ratio calculation following NS/ISDN instillation. No significant differences between the NS $(3 \mathrm{ml} / \mathrm{kg})$ and ISDN ( $3 \mathrm{ml} / \mathrm{kg}$ ) groups were observed for the following parameters: Wet lung weight to BW $(0.71 \pm 0.06$ vs. $0.73 \pm 0.08 \%)$, dry lung weight to BW $(0.16 \pm 0.04$ vs. $0.13 \pm 0.06 \%)$ and dry to wet lung weight (21.82 \pm 5.10 vs. $17.52 \pm 6.62 \%$; Fig. $2 \mathrm{E})$.

Plasma NO concentration assessment following NS/ISDN intratracheal instillation. Fig. 2F shows that ISDN intratracheal instillation increased plasma NO levels. Following ISDN instillation, plasma NO levels were rapidly and significantly increased $(\mathrm{P}=0.017)$, as shown by analysis of the blood sample taken immediately after instillation. The aforementioned results demonstrated that the changes in MPAP and MAP induced by $3 \mathrm{mg} / \mathrm{kg}$ ISDN lasted for the $15 \mathrm{~min}$ of administration. However, plasma NO levels in the ISDN (3 mg/kg) group were higher than those in NS rats even following 15 min of ISDN instillation (Fig. 2F). The onset of action of ISDN after inhalation was very rapid ( $<1 \mathrm{~min}$ ) but lasted for $5 \mathrm{~min}$.

Echocardiogram and hemodynamic measurement in rats 14 days after MI. LV enlargement was attenuated in the MI-ISDN group on day 15 since LVIDd and LV Vol d were markedly reduced compared with those in the MI-NS group. LV systolic function was also improved in the MI-ISDN group as LVIDs and LV Vol s were decreased in comparison with those in the MI-NS group, and LVEF\% and FS\% were significantly increased. LVAWd and LVAWs in rats of the MI-ISDN group were thicker compared with those in the MI-NS group, which may be associated with a reduction in the MI area. Moreover, LVPWd and LVPWs were thinner in rats treated with ISDN inhalation compared with those receiving NS inhalation, suggesting that myocardium hypertrophy occurred in the LV posterior wall and was improved by ISDN inhalation (Table I).

Hemodynamic variables (Table II) further suggested that LV systolic function was improved by ISDN inhalation, since $\mathrm{LV}+\mathrm{dp} / \mathrm{dt}_{\max }$ was significantly higher in the MI-ISDN group compared with the MI-NS group. LV diastolic function was improved in the MI-ISDN group, as shown by the LVEDP and $\mathrm{LV}-\mathrm{dp} / \mathrm{dt}_{\max }$ measurements. The diastolic function improvement may be associated with attenuation of LV hypertrophy, as shown by the echocardiogram data.

Systolic pulmonary pressure, as estimated by RV systolic pressure (RVSP), was elevated in the MI-NS and MI-ISDN groups compared with the sham group. ISDN inhalation reduced RSVP and improved RV systolic and diastolic functions, as characterized by improvements of $\mathrm{RV} \pm \mathrm{dp} / \mathrm{dt}_{\max }$, RVEDP and CVP, in comparison with NS inhalation. MAP was lower in the MI-ISDN group compared with the MI-NS and sham groups, but the difference was not statistically significant. The HR in the MI-NS group was faster than those in the MI-ISDN and sham groups (Table II).

$H W / B W \%, L V / B W \%$ and $R V / B W \%$ evaluations. The calculated values of $\mathrm{HW} / \mathrm{BW} \%, \mathrm{LV} / \mathrm{BW} \%$ and $\mathrm{RV} / \mathrm{BW} \%$ indicated that LV and RV hypertrophy occurred in rats following MI $(\mathrm{P}<0.05$ MI-NS vs. sham) and was improved by ISDN inhalation ( $\mathrm{P}<0.05$ MI-ISDN vs. MI-NS; Fig. 3A).

MI area \% and VEGF-positive area \% estimation. The MI area \%, as estimated by Masson staining, was observed to be decreased by ISDN inhalation in rats following MI, although not to as low a level as that in the sham group $(\mathrm{P}<0.05$ among the three groups; Fig. 3B-D). In addition, the 
A

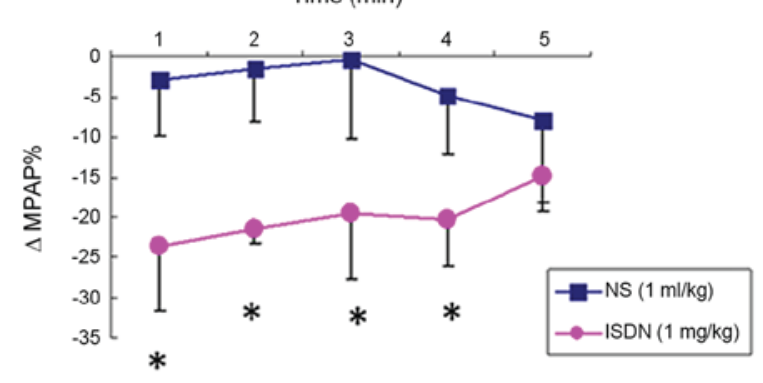

C

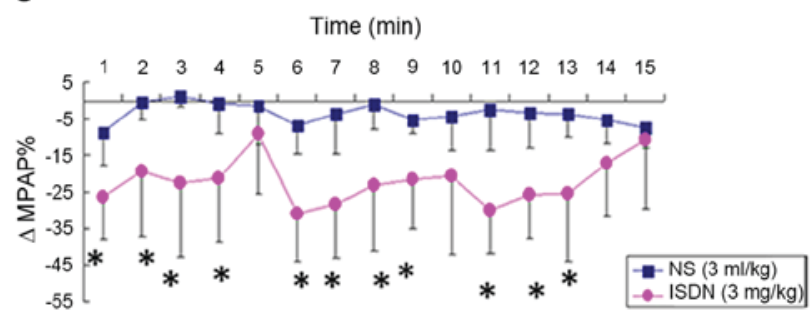

B

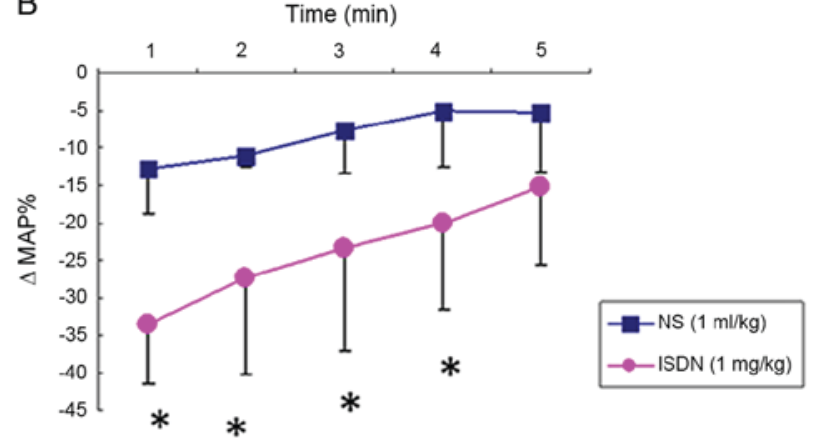

D

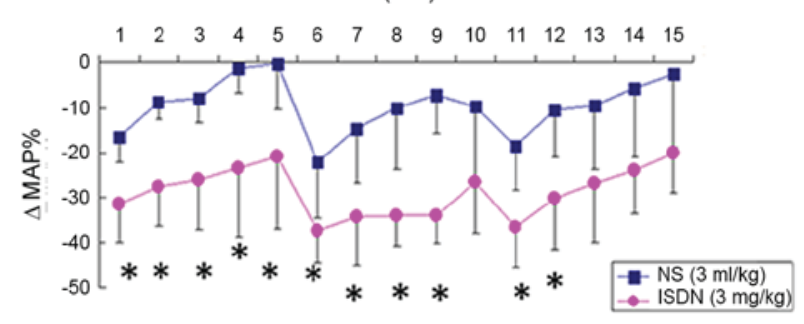

E
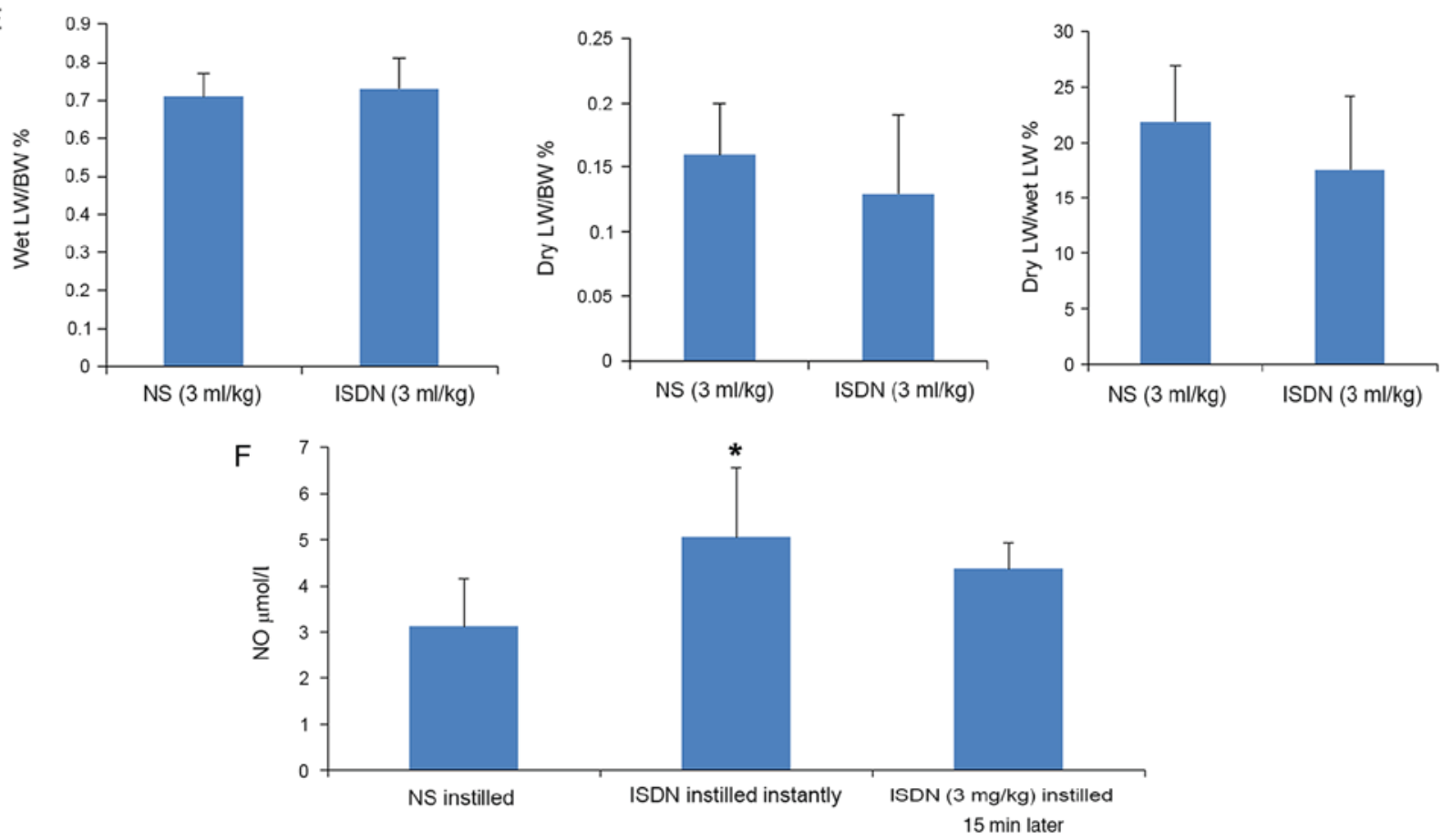

Figure 2. $\triangle \mathrm{MPAP}, \triangle \mathrm{MAP}$, lung weight and NO in the NS and ISDN groups. Differences in (A) $\triangle \mathrm{MPAP} \%$ and (B) $\triangle \mathrm{MAP} \%$ were detected following the intratracheal instillation of $1 \mathrm{mg} / \mathrm{kg}$ ISDN vs. $1 \mathrm{ml} / \mathrm{kg} \mathrm{NS}$ in MI rats. Changes in (C) $\triangle \mathrm{MPAP} \%$ and (D) $\triangle \mathrm{MAP} \%$ were detected following the intratracheal instillation of $3 \mathrm{mg} / \mathrm{kg}$ ISDN vs. $3 \mathrm{ml} / \mathrm{kg}$ NS in MI rats. "P<0.05 vs. the NS group; $\mathrm{n}=5 / \mathrm{group}$. (E) Wet LW/BW\%, dry LW/BW\% and dry LW/BW\% following the intratracheal instillation of $3 \mathrm{ml} / \mathrm{kg}$ NS or $3 \mathrm{mg} / \mathrm{kg}$ ISDN. (F) Plasma NO levels following NS/ISDN instillation. "P<0.05 vs. the NS group; $\mathrm{n}=5 /$ group. $\triangle \mathrm{MPAP} \%$, percentage change in mean pulmonary arterial pressure; $\triangle \mathrm{MAP} \%$, percentage change in mean arterial pressure; NO, nitric oxide; NS, normal saline; ISDN, isosorbide dinitrate; MI, myocardial infarction; LW, lung weight; BW, body weight.

VEGF-positive area $\%$ at the border of the MI region was significantly increased $(\mathrm{P}=0.012)$ by ISDN inhalation, as shown in Fig. 3E.

Levels of neurohormonal factors. Plasma levels of epinephrine and norepinephrine, as shown in Fig. 4A, were reduced by inhaled ISDN in rats following MI. The differences in norepinephrine levels among the groups were in accordance with the differences of HR among the groups.
Plasma levels of BNP were significantly elevated in the MI-NS group compared with the sham group $(786.78 \pm 161.10$ vs. $644.02 \pm 102.10 \mathrm{pg} / \mathrm{ml}, \mathrm{P}=0.015)$, and ISDN inhalation decreased the BNP levels in the MI rats $(670.65 \pm 93.30$ vs. $786.78 \pm 161.10 \mathrm{pg} / \mathrm{ml}, \mathrm{P}=0.044)$. These findings suggest that ISDN inhalation ameliorated LV remodeling and cardiac dysfunction (Fig. 4B).

Inhaled ISDN, however, only had slight effect on the plasma concentration of angiotensin II $(35.81 \pm 3.67$ 
Table I. Echocardiogram measurements.

\begin{tabular}{lcrr}
\hline Variables & MI-NS & MI-ISDN & Sham \\
\hline LVIDd (mm) & $7.63 \pm 1.03^{\mathrm{a}, \mathrm{b}}$ & $6.07 \pm 1.29^{\mathrm{b}}$ & $5.03 \pm 0.65$ \\
LVIDs $(\mathrm{mm})$ & $6.26 \pm 1.38^{\mathrm{a}, \mathrm{b}}$ & $3.75 \pm 1.99^{\mathrm{b}}$ & $1.76 \pm 1.01$ \\
LV Vol d $(\mu \mathrm{l})$ & $317.03 \pm 91.00^{\mathrm{a}, \mathrm{b}}$ & $195.24 \pm 93.68^{\mathrm{b}}$ & $122.64 \pm 37.58$ \\
LV Vol s $(\mu \mathrm{l})$ & $209.74 \pm 102.15^{\mathrm{a}, \mathrm{b}}$ & $83.54 \pm 94.22$ & $14.67 \pm 25.18$ \\
LVPWd $(\mathrm{mm})$ & $1.92 \pm 0.34^{\mathrm{a}, \mathrm{b}}$ & $1.56 \pm 0.22^{\mathrm{b}}$ & $1.32 \pm 0.18$ \\
LVPWs $(\mathrm{mm})$ & $2.50 \pm 0.20$ & $2.34 \pm 0.28$ & $2.32 \pm 0.34$ \\
LVAWd $(\mathrm{mm})$ & $1.00 \pm 0.21^{\mathrm{a}, \mathrm{b}}$ & $1.45 \pm 0.45$ & $1.55 \pm 0.14$ \\
LVAWs $(\mathrm{mm})$ & $1.14 \pm 0.36^{\mathrm{a}, \mathrm{b}}$ & $2.01 \pm 0.83^{\mathrm{b}}$ & $2.94 \pm 0.35$ \\
LVEF\% & $36.76 \pm 15.14^{\mathrm{a}, \mathrm{b}}$ & $66.35 \pm 24.73^{\mathrm{b}}$ & $90.73 \pm 12.93$ \\
FS\% & $18.79 \pm 8.55^{\mathrm{a}, \mathrm{b}}$ & $41.09 \pm 20.21^{\mathrm{b}}$ & $66.32 \pm 14.25$ \\
\end{tabular}

${ }^{\mathrm{a}} \mathrm{P}<0.05$ vs. the MI-ISDN group; ${ }^{\mathrm{b}} \mathrm{P}<0.05$ vs. the sham group. LVIDd, left ventricular internal diameter at end-diastole; LVIDs, left ventricular internal diameter at end-systole; LV Vol d, left ventricular volume at end-diastole; LV Vol s, left ventricular volume at end-systole; LVPWd, left ventricular post wall diameter at end-diastole; LVPWs, left ventricular post wall diameter at end-systole; LVAWd, left ventricular anterior wall diameter at end-diastole; LVAWs, left ventricular anterior wall diameter at end-systole; LVEF, left ventricular ejection fraction; FS, fraction shortening.

Table II. Hemodynamic variables.

\begin{tabular}{lccc}
\hline Variables & MI-NS & MI-ISDN & Sham \\
\hline MAP $(\mathrm{mmHg})$ & $146.54 \pm 24.06$ & $134.06 \pm 28.96$ & $149.70 \pm 29.45$ \\
HR $(\mathrm{bpm})$ & $426 \pm 65^{\mathrm{a}}$ & $397 \pm 41$ & $358 \pm 64$ \\
$\mathrm{LVSP}(\mathrm{mmHg})$ & $171.35 \pm 24.50$ & $157.75 \pm 35.47$ & $166.70 \pm 20.81$ \\
$\mathrm{LVEDP}(\mathrm{mmHg})$ & $11.06 \pm 5.10^{\mathrm{a}, \mathrm{b}}$ & $5.85 \pm 3.50$ & $3.82 \pm 0.70$ \\
$\mathrm{LV}+\mathrm{dp} / \mathrm{dt}_{\max }(\mathrm{mmHg} / \mathrm{sec})$ & $5,284.52 \pm 621.76^{\mathrm{a}, \mathrm{b}}$ & $7,998.46 \pm 2,761.47$ & $6,837.68 \pm 424.74$ \\
$\mathrm{LV}-\mathrm{dp} / \mathrm{dt}_{\max }(\mathrm{mmHg} / \mathrm{sec})$ & $3,558.97 \pm 842.38^{\mathrm{a}, \mathrm{b}}$ & $5,322.14 \pm 1,325.26$ & $4,837.96 \pm 868.72$ \\
$\mathrm{RVSP}(\mathrm{mmHg})$ & $47.72 \pm 5.05^{\mathrm{a}, \mathrm{b}}$ & $30.76 \pm 16.94$ & $23.58 \pm 6.18$ \\
$\mathrm{RVEDP}(\mathrm{mmHg})$ & $7.63 \pm 1.44^{\mathrm{a}, \mathrm{b}}$ & $2.09 \pm 4.38$ & $0.26 \pm 3.51$ \\
$\mathrm{RV}+\mathrm{dp} / \mathrm{dt}_{\max }(\mathrm{mmHg} / \mathrm{sec})$ & $2,902.55 \pm 485.22^{\mathrm{a}, \mathrm{b}}$ & $2,375.61 \pm 421.41$ & $1,789.64 \pm 444.28$ \\
$\mathrm{RV}-\mathrm{dp} / \mathrm{dt}_{\max }(\mathrm{mmHg} / \mathrm{sec})$ & $2,173.92 \pm 343.32^{\mathrm{a}, \mathrm{b}}$ & $1,621.45 \pm 301.87$ & $5.44 \pm 1.25$ \\
$\mathrm{CVP}\left(\mathrm{cmH}_{2} \mathrm{O}\right)$ & $9.39 \pm 1.63^{\mathrm{a}, \mathrm{b}}$ & $6.91 \pm 2.51$ & $2.13 \pm 407.53$ \\
\hline
\end{tabular}

${ }^{\mathrm{a}} \mathrm{P}<0.05$ vs. the sham group; ${ }^{\mathrm{b}} \mathrm{P}<0.05$ vs. the MI-ISDN group. MAP, mean arterial pressure; HR, heart rate; LVSP, left ventricular systolic pressure; LVEDP, left ventricular end-diastolic pressure; $\mathrm{LV}+\mathrm{dp} / \mathrm{dt}_{\max }$, the maximum increase rate of left ventricular $\mathrm{pressure;} \mathrm{L}-\mathrm{dp} / \mathrm{dt}_{\max }$, the maximum dropping rate of left ventricular pressure; RVSP, right ventricular systolic pressure; RVEDP, right ventricular end-diastolic pressure; $\mathrm{RV}+\mathrm{dp} / \mathrm{dt}_{\max }$, the maximum increase rate of right ventricular pressure; $\mathrm{RV}-\mathrm{dp} / \mathrm{dt}_{\max }$, the maximum dropping rates of right ventricular pressure; CVP, central venous pressure.

vs. $42.41 \pm 12.00 \mathrm{pg} / \mathrm{ml}$ in the MI-ISDN and MI-NS groups, respectively, $\mathrm{P}=0.331$; Fig. $4 \mathrm{C}$ ).

\section{Discussion}

PH due to LHD is associated with poor outcome and there is a lack of supportive management for excessive vasoconstriction or remodeling of the pulmonary artery. Available guidelines do not recommend treatment for the direct reduction of pulmonary pressure due to LHD, since this kind of intervention could potentially dilate the pulmonary vessels and induce pulmonary edema (4). Nevertheless, benefits from sildenafil, a phosphodiesterase-5 inhibitor have been reported in such patients (27). These findings suggest that dilation of the pulmonary artery as well as systemic vessels with sildenafil may lead to unloading of the left and right ventricles and decreased pulmonary congestion (6). Therefore, the present study investigated the benefits of ISDN inhalation on pulmonary pressure and ventricular remodeling in a rat model of HF following MI. Results demonstrated that intratracheal ISDN led to significantly greater $\triangle \mathrm{MPAP} \%$ and $\triangle \mathrm{MAP} \%$ than NS, without pulmonary edema. These changes were associated with increased plasma NO levels. ISDN inhalation for 14 days reduced MI size and alleviated LV and RV remodeling following MI. These hemodynamic and morphological improvements were associated with decreased plasma levels 

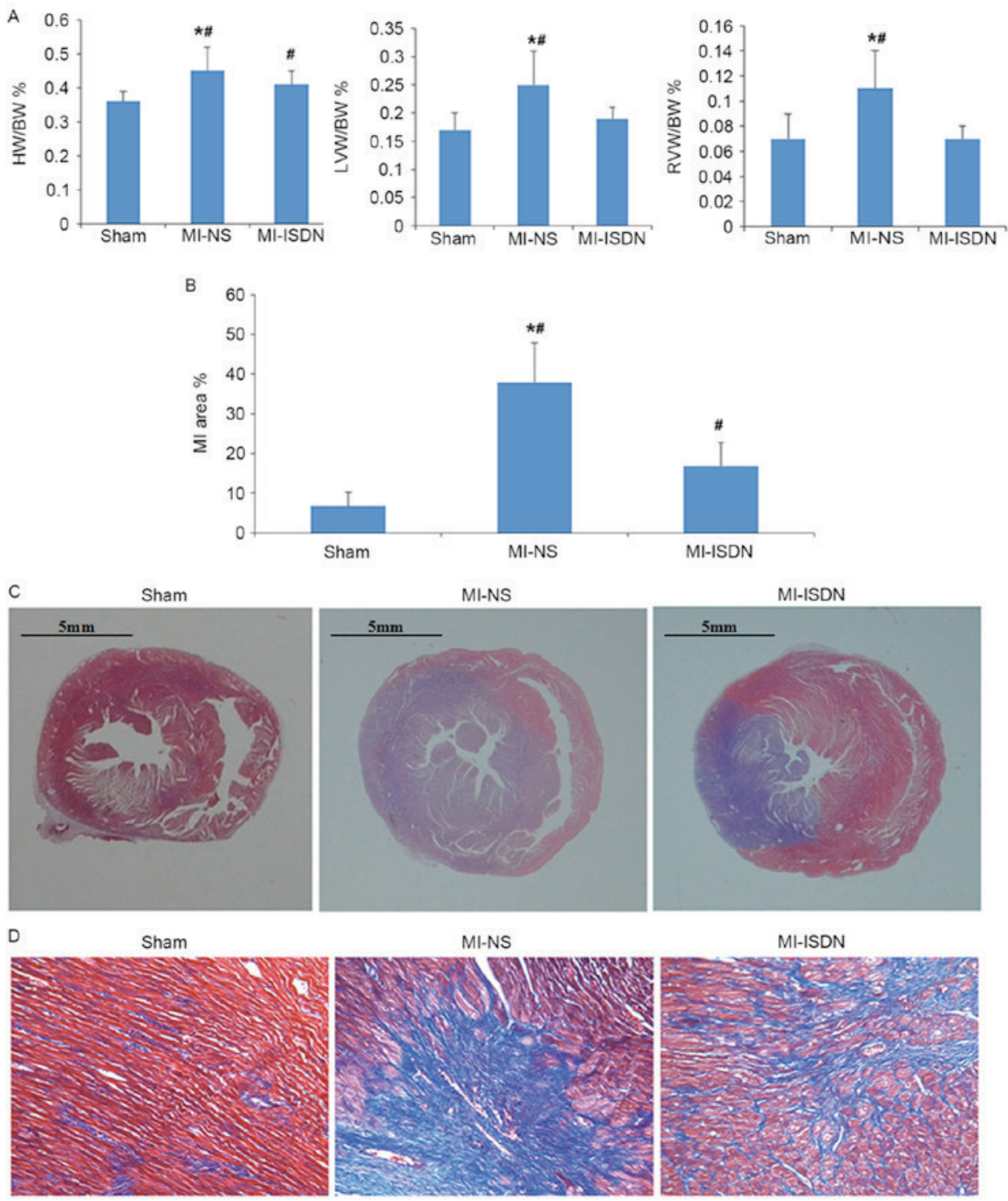

MI-NS

MI-ISDN
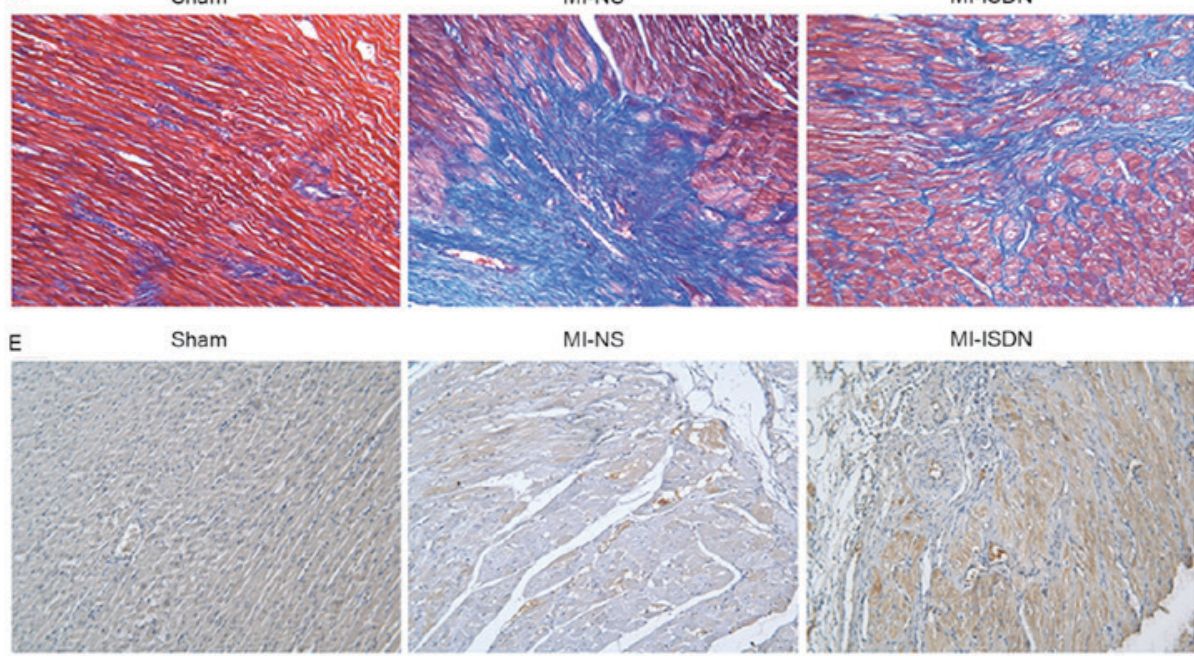

MI-NS

MI-ISDN
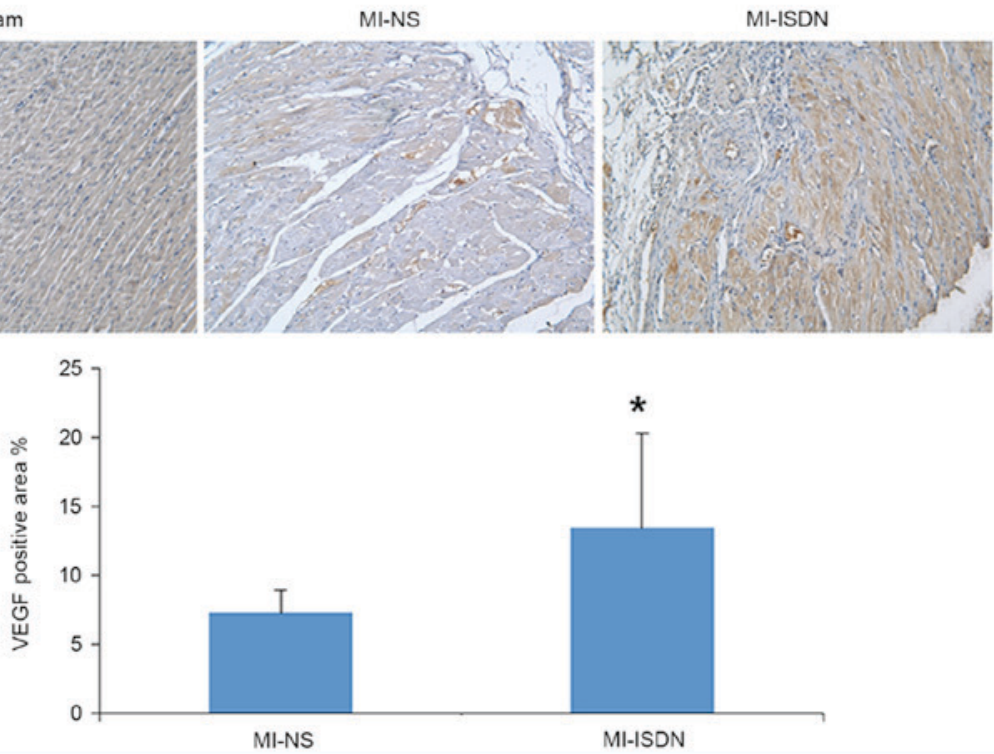

Figure 3. Parameters of heart damage among the three groups. (A) HW/BW\%, LVW/BW\% and RVW/BW\% of rats on day 15 . ${ }^{*} \mathrm{P}<0.05$ vs. MI-ISDN; ${ }^{~} \mathrm{P}<0.05$ vs. sham; $\mathrm{n}=10$ /group. (B) MI area \% in rats on day 15 . ${ }^{*} \mathrm{P}<0.05$ vs. MI-ISDN; ${ }^{\#} \mathrm{P}<0.05$ vs. sham; $\mathrm{n}=10 / \mathrm{group}$. (C) Myocardium tissues with Masson staining. The blue area indicates collagen proliferation following myocardial necrosis. Scale bar, 5 mm. (D) Myocardium tissues with Masson staining. The blue area indicates collagen proliferation following myocardial necrosis (magnification, x200). (E) VEGF positive area in each group. The brown area indicates areas of VEGF expression on the border of the MI region (magnification, x200). * $\mathrm{P}<0.05$ vs. MI-NS; n=10/group. HW, heart weight; BW, body weight; LVW, left ventricular weight; RVW, right ventricular weight; MI, myocardial infarction; ISDN, isosorbide dinitrate; NS, normal saline; VEGF, vascular endothelial growth factor. 


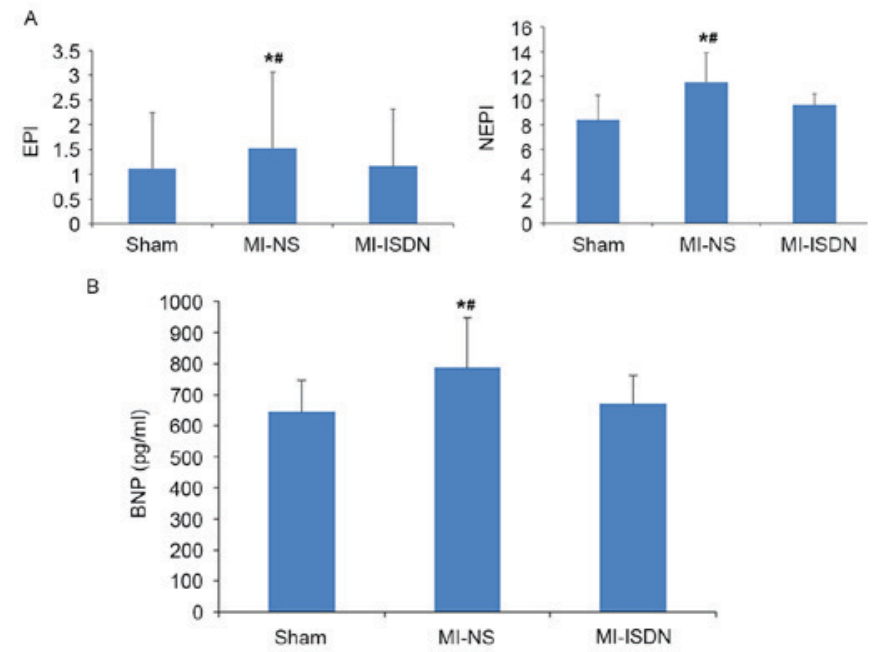

c

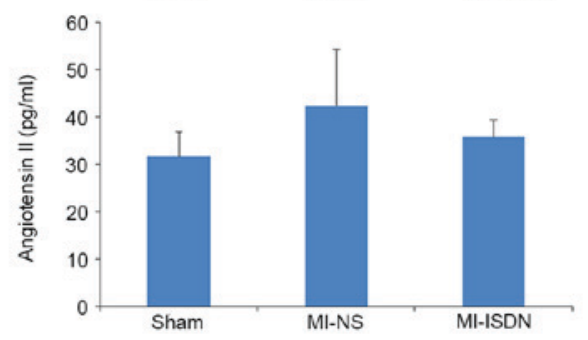

Figure 4. Levels of neurohormonal factors. (A) Plasma levels of EPI and NEPI on day 15 . ${ }^{*} \mathrm{P}<0.05$ vs. MI+ISDN; ${ }^{~} \mathrm{P}<0.05$ vs. sham; $\mathrm{n}=10$ /group. (B) Plasma levels of BNP among the groups. ${ }^{*} \mathrm{P}<0.05$ vs. MI-ISDN; ${ }^{*} \mathrm{P}<0.05$ vs. sham; $n=10$ /group. (C) Plasma levels of angiotensin II among the groups. MI, myocardial infarction; NS, normal saline; ISDN, isosorbide dinitrate; EPI, epinephrine; NEPI, norepinephrine; BNP, B-type natriuretic peptide.

of epinephrine, norepinephrine and BNP, and an increased VEGF positive area at the border of the MI region.

Experimental studies conducting morphometric analysis $\sim 4$ weeks after coronary ligation are not rare; however, significant hemodynamic and structural changes can be detected only 2 weeks after MI (28). Hence, the present study assessed hemodynamics $\leq 15$ days after coronary ligation. The LVW/BW\% was elevated in the MI-NS group, which indicated that LV hypertrophy occurred in rats following MI. In addition, LV hypertrophy was mainly present in the posterior wall, remote from the infarcted area, according to echocardiogram measurements. Thus, LV diastolic dysfunction in rats following MI, assessed by increased LVEDP and decreased LV-dp/dt ${ }_{\max }$, may be a consequence of LV hypertrophy, or LV eccentric remodeling with LV enlargement. In the present study, an ISDN dose restricted to $3 \mathrm{mg} / \mathrm{kg}$ inhaled for 15 min every day for 14 days was demonstrated to be effective in improving ventricular parameters. In addition, echocardiogram and hemodynamic measurements indicated that ISDN inhalation improved LV and RV remodeling and function. LV/BW\% and LVPWd were similar between the MI-ISDN and sham groups. Thus, amelioration of LV hypertrophy by ISDN inhalation resulted in improvements in LV diastolic function, which was reflected by decreased LVEDP and increased LV-dp/dt $t_{\max }$.

$\mathrm{RV}$ dysfunction is known to be a complication of MI with or without PH in experimental and clinical studies $(3,29)$. In the present study, RV hypertrophy with systolic and diastolic dysfunction was detected in rats of the MI-NS group. Increased
RVSP may be a response to the increased afterload of RV due to elevated LVEDP and potentially increased pulmonary arterial resistance. Therefore, direct pulmonary pressure reduction with ISDN inhalation could lead to RV unloading and prove beneficial to RV remodeling. In addition, improvement of LV function, such as decline of LVEDP, may be another mechanism of RV remodeling reversion observed in the present study. The precise mechanism of RV remodeling following LV MI remains unclear. However, the results of the present study suggest that ISDN inhalation could be a promising therapy for RV dysfunction following MI.

Excess pressure or volume load, neurohormonal activation and progressive myocardial remodeling with $\mathrm{LV}$ wall stress may be detrimental to cardiac cycles and result in ventricular dilation and cardiac dysfunction following MI (30). Pure volume and pressure unloading, achieved by implantation of an LV assist device (LVAD), have been demonstrated to be efficient in reversing ventricular remodeling in patients with HF (31). This remodeling improvement has been indicated to be associated with a normalization of circulating neurohormones, including epinephrine and norepinephrine (32). In view of these findings, LV volume and pressure unloading with ISDN inhalation may directly disrupt detrimental cycles and alleviate ventricular remodeling. Sympathetic efferent neuronal activity is increased in patients with HF (33). Excessive exposure of the myocardium to norepinephrine has been shown to result in worsening HF with downregulation of $\alpha$ and $\beta$ receptors, increased oxygen consumption, and loss of contractile reserve (34). In addition, it has been reported that elevated sympathetic activity causes enlargement of the MI area and exacerbates myocardial remodeling (25). Thus, a reduction in the plasma levels of epinephrine and norepinephrine by ISDN inhalation in rats following MI is likely to be beneficial to $\mathrm{LV}$ remodeling. Therefore, $\mathrm{LV}$ and $\mathrm{RV}$ unloading by ISDN inhalation may establish a beneficial cycle associated with reduced neurohormonal activation, finally improving LV and RV remodeling.

Reduction of the area of MI with ISDN inhalation may provide a great contribution to LV morphological alterations and systolic function improvements. The present study suggests some possible mechanisms. First, an increase in the VEGF-positive area at the border of the MI region following ISDN inhalation may reduce the MI area. VEGF potentially induces angiogenesis under ischemic conditions and plays a key role in repair of the myocardium following MI (35). Moreover, it has been reported that NO regulates VEGF expression and mediates VEGF-induced endothelial cell proliferation and migration (36). Therefore, regulation of VEGF distribution in the ischemic area by ISDN, a NO donor, may promote myocardium viability following MI. However, it remains to be elucidated whether the effects on VEGF expression are caused only by NO released from ISDN or by the NO-dependent actions of ISDN or its derivatives (37).

Secondly, hemodynamic improvement may itself minimize MI size. Sun et al (38) observed that the reversion of unstable hemodynamics supported by LVAD decreased LV volume and wall stress, relieved LV remodeling, and preserved LV function due to minimization of the MI size in a swine model of acute MI. Similar findings were noted in the present study since LV and RV unloading by ISDN inhalation improved the 
hemodynamics in the systematic and pulmonary circulations This type of hemodynamic support may directly contribute to a reduction in MI size.

Third, increased plasma NO levels following the intratracheal instillation of ISDN indicated that the pharmacological effects of ISDN were not limited to the pulmonary circulation. In addition, relatively persistent increased NO levels in the peripheral blood further indicated that the protective effects of ISDN may go beyond hemodynamic improvements. It may be assumed that reduction of the MI area is regulated by the NO-soluble guanylyl cyclase-cyclic guanosine monophosphate axis, as previously indicated (14). However, additional studies are necessary to address the mechanisms properly.

There are some limitations to this study. Firstly, it was not clear whether high doses of ISDN inhalation lasting for a longer time could produce greater effects on ventricle remodeling and cardiac function. In addition, the mechanism by which MI size is reduced by ISDN inhalation requires further investigation.

In conclusion, intratracheal instillation of ISDN has been demonstrated to improve the hemodynamics of the pulmonary and systemic circulation in MI rats without inducing pulmonary edema. These benefits were associated with increased plasma NO levels. ISDN instillation/inhalation for 14 days decreased MI area and alleviated LV and RV remodeling in rats following MI. The hemodynamic and morphological improvements were associated with decreased plasma levels of epinephrine, norepinephrine and BNP, and an increased VEGF positive area at the border of the MI region.

\section{Acknowledgements}

The authors would like to thank Mr. Hao Wang and Mr. Zhonghua $\mathrm{Wu}$ for their contribution to the establishment of the rat MI model and immunohistochemical measurements. This study was supported by the Discovery Fund from the Chinese Medical Doctor Association (DFCMDA201309).

\section{References}

1. Kumar A and Cannon CP: Acute coronary syndromes: Diagnosis and management, part I. Mayo Clin Proc 84: 917-938, 2009.

2. Barnett $\mathrm{CF}$ and De Marco T: Pulmonary hypertension associated with left-sided heart disease. Heart Fail Clin 8: 447-459, 2012.

3. Toldo S, Bogaard HJ, Van Tassell BW, Mezzaroma E, Seropian IM, Robati R, Salloum FN, Voelkel NF and Abbate A: Right ventricular dysfunction following acute myocardial infarction in the absence of pulmonary hypertension in the mouse. PLoS One 6: e18102, 2011.

4. Galiè N, Hoeper MM, Humbert M, Torbicki A, Vachiery JL, Barbera JA, Beghetti M, Corris P, Gaine S, Gibbs JS, et al: Guidelines for the diagnosis and treatment of pulmonary hypertension: The task force for the diagnosis and treatment of pulmonary hypertension of the european society of cardiology (ESC) and the european respiratory society (ERS), endorsed by the international society of heart and lung transplantation (ISHLT). Eur Heart J 30: 2493-2537, 2009.

5. Gajarsa JJ and Kloner RA: Left ventricular remodeling in the post-infarction heart: A review of cellular, molecular mechanisms, and therapeutic modalities. Heart Fail Rev 16: 13-21, 2011.

6. Lundgren $\mathrm{J}$ and Rådegran G: Pathophysiology and potential treatments of pulmonary hypertension due to systolic left heart failure. Acta Physiol (Oxf) 211: 314-333, 2014.

7. Vachiéry JL, Adir Y, Barberà JA, Champion H, Coghlan JG, Cottin V, De Marco T, Galiè N, Ghio S, Gibbs JS, et al: Pulmonary hypertension due to left heart diseases. J Am Coll Cardiol 62 (25 Suppl): D100-D108, 2013.
8. Galie N, Humbert M, Vachiery JL, Gibbs S, Lang I, Torbicki A, Simonneau G, Peacock A, Vonk Noordegraaf A, Beghetti M, et al: $2015 \mathrm{ESC} / \mathrm{ERS}$ Guidelines for the diagnosis and treatment of pulmonary hypertension. The Joint Task Force for the Diagnosis and Treatment of Pulmonary Hypertension of the European Society of Cardiology (ESC) and the European Respiratory Society (ERS). Eur Respir J 46: 903-975, 2015.

9. Ignarro LJ: Nitric oxide as a unique signaling molecule in the vascular system: A historical overview. J Physiol Pharmacol 53: 503-514, 2002.

10. Ignarro LJ, Buga GM, Wood KS, Byrns RE and Chaudhuri G: Endothelium-derived relaxing factor produced and released from artery and vein is nitric oxide. Proc Natl Acad Sci USA 84: 9265-9269, 1987.

11. Napoli C and Ignarro LJ: Nitric oxide and pathogenic mechanisms involved in the development of vascular diseases. Arch Pharm Res 32: 1103-1108, 2009.

12. Blum M, Yachnin T, Wollman Y, Chernihovsky T, Peer G, Grosskopf I, Kaplan E, Silverberg D, Cabili S and Iaina A: Low nitric oxide production in patients with chronic renal failure. Nephron 79: 265-268, 1998.

13. Schmidt RJ and Baylis C: Total nitric oxide production is low in patients with chronic renal disease. Kidney Int 58: 1261-1266, 2000.

14. Neye N, Enigk F, Shiva S, Habazettl H, Plesnila N, Kuppe H, Gladwin MT and Kuebler WM: Inhalation of NO during myocardial ischemia reduces infarct size and improves cardiac function. Intensive Care Med 38: 1381-1391, 2012.

15. Ghofrani HA, Galiè N, Grimminger F, Grünig E, Humbert M, Jing ZC, Keogh AM, Langleben D, Kilama MO, Fritsch A, et al: Riociguat for the treatment of pulmonary arterial hypertension. N Engl J Med 369: 330-340, 2013

16. Dejam A, Hunter CJ, Tremonti C, Pluta RM, Hon YY, Grimes G, Partovi K, Pelletier MM, Oldfield EH, Cannon RO III, et al: Nitrite infusion in humans and nonhuman primates: Endocrine effects, pharmacokinetics, and tolerance formation. Circulation 116: 1821-1831, 2007.

17. Puikuan K, Chunyu Z, Jin F, Chaoshu T and Junbao D: Inhalation of nebulized nitroglycerin, a nitric oxide donor, for the treatment of pulmonary hypertension induced by high pulmonary blood flow. Heart Vessels 21: 169-179, 2006.

18. Xia HP, Huang GY, Zhu JX and Sun B: Effect of inhalation of nebulized NO donor substance on acute hypoxic lung injury in newborn piglets. Chin Med J (Engl) 121: 1622-1626, 2008.

19. Horinaka S, Kobayashi N, Yagi H, Mori Y and Matsuoka H: Nicorandil but not ISDN upregulates endothelial nitric oxide synthase expression, preventing left ventricular remodeling and degradation of cardiac function in Dahl salt-sensitive hypertensive rats with congestive heart failure. J Cardiovasc Pharmacol 47: 629-635, 2006.

20. Cohn JN, Tam SW, Anand IS, Taylor AL, Sabolinski ML and Worcel M; A-HeFT Investigators: Isosorbide dinitrate and hydralazine in a fixed-dose combination produces further regression of left ventricular remodeling in a well-treated black population with heart failure: Results from A-HeFT. J Card Fail 13: 331-339, 2007.

21. National Research Council (US): Guide for the Care and Use of Laboratory Animals, 8th edition. National Academies Press (US), Washington, DC, 2011.

22. Zornoff LA, Paiva SA, Minicucci MF and Spadaro J: Experimental myocardium infarction in rats: Analysis of the model. Arq Bras Cardiol 93: 434-440, 426-432, 2009 (In English, Portuguese, Spanish).

23. Horie M, Yoshiura Y, Izumi H, Oyabu T, Tomonaga T, Okada T, Lee BW, Myojo T, Kubo M, Shimada M and Morimoto Y: Comparison of the pulmonary oxidative stress caused by intratracheal instillation and inhalation of $\mathrm{NiO}$ nanoparticles when equivalent amounts of $\mathrm{NiO}$ are retained in the lung. Antioxidants (Basel) 5: pii: E4, 2016.

24. Stefanon I, Valero-Muñoz M, Fernandes AA, Ribeiro RF Jr, Rodríguez C, Miana M, Martínez-González J, Spalenza JS, Lahera V, Vassallo PF and Cachofeiro V: Left and right ventricle late remodeling following myocardial infarction in rats. PLoS One 8: e64986, 2013.

25. Shi S, Liang J, Liu T, Yuan X, Ruan B, Sun L, Tang Y, Yang B, $\mathrm{Hu}$ D and Huang C: Depression increases sympathetic activity and exacerbates myocardial remodeling after myocardial infarction: Evidence from an animal experiment. PLoS One 9: e101734, 2014. 
26. Zhao Q, Sun C, Xu X, Zhou J, Wu Y, Tian Y, Yuan Z and Liu Z: $\mathrm{CD} 34^{+}$cell mobilization and upregulation of myocardial cytokines in a rabbit model of myocardial ischemia. Int J Cardiol 152: 18-23, 2011.

27. Reichenbach A, Al-Hiti H, Malek I, Pirk J, Goncalvesova E, Kautzner J and Melenovsky V: The effects of phosphodiesterase 5 inhibition on hemodynamics, functional status and survival in advanced heart failure and pulmonary hypertension: A case-control study. Int J Cardiol 168: 60-65, 2013.

28. Jasmin JF, Mercier I, Hnasko R, Cheung MW, Tanowitz HB, Dupuis $\mathrm{J}$ and Lisanti MP: Lung remodeling and pulmonary hypertension after myocardial infarction: Pathogenic role of reduced caveolin expression. Cardiovasc Res 63: 747-755, 2004.

29. Van Tassell BW, Bhardwaj HL, Grizzard JD, Kontos MC, Bogaard H, Gomez-Arroyo J, Toldo S, Mezzaroma E, Voelkel NF and Abbate A: Right ventricular systolic dysfunction in patients with reperfused ST-segment elevation acute myocardial infarction. Int J Cardiol 155: 314-316, 2012.

30. Abd-Elmoniem KZ, Tomas MS, Sasano T, Soleimanifard S, Vonken EJ, Youssef A, Agarwal H, Dimaano VL, Calkins H, Stuber M, et al: Assessment of distribution and evolution of mechanical dyssynchrony in a porcine model of myocardial infarction by cardiovascular magnetic resonance. J Cardiovasc Magn Reson 14: 1, 2012.

31. Drakos SG, Kfoury AG, Selzman CH, Verma DR, Nanas JN, Li DY and Stehlik J: Left ventricular assist device unloading effects on myocardial structure and function: Current status of the field and call for action. Curr Opin Cardiol 26: 245-255, 2011.
32. George RS, Birks EJ, Cheetham A, Webb C, Smolenski RT, Khaghani A, Yacoub MH and Kelion A: The effect of long-term left ventricular assist device support on myocardial sympathetic activity in patients with non-ischaemic dilated cardiomyopath. Eur J Heart Fail 15: 1035-1043, 2013.

33. Samson R, Baydoun H, Jaiswal A and Le Jemtel TH: Cardiac adrenergic nervous system and left ventricular remodeling. Am J Med Sci 350: 321-326, 2015.

34. Haider N, Baliga RR, Chandrashekhar Y and Narula J: Adrenergic excess, hNET1 down-regulation, and compromised mIBG uptake in heart failure poverty in the presence of plenty. JACC Cardiovasc Imaging 3: 71-75, 2010.

35. Cai M, Ren L, Yin X, Guo Z, Li Y, He T, Tang Y, Long T, Liu Y, Liu G, et al: PET monitoring angiogenesis of infarcted myocardium after treatment with vascular endothelial growth factor and bone marrow mesenchymal stem cells. Amino Acids 48: 811-820, 2016.

36. Zhang R, Wang L, Zhang L, Chen J, Zhu Z, Zhang Z and Chopp M: Nitric oxide enhances angiogenesis via the synthesis of vascular endothelial growth factor and cGMP after stroke in the rat. Circ Res 92: 308-313, 2003.

37. Rammos C, Luedike P, Hendgen-Cotta U and Rassaf T: Potential of dietary nitrate in angiogenesis. World J Cardiol 7: 652-657, 2015.

38. Sun X, Li J, Zhao W, Lu S, Guo C, Lai H and Wang C: Early assistance with left ventricular assist device limits left ventricular remodeling after acute myocardial infarction in a swine model. Artif Organs 40: 243-251, 2016. 